\title{
Analysis and Development of a Digital Radar Flaw Detector for Robotic Defect Detection Systems
}

\author{
V. S Potylitsyn \\ Institute of Radio Technology \\ Siberian Federal University \\ Krasnoyarsk, Russia \\ markuss86@mail.ru
}

\author{
E. A Kokhonkova \\ Military Engineering Institute \\ Siberian Federal University \\ Krasnoyarsk, Russia \\ kokhonkova@yandex.ru
}

\author{
V. V. Romanov \\ Institute of Radio Technology \\ Siberian Federal University \\ Krasnoyarsk, Russia \\ slava-romanov-98@mail.ru
}

\begin{abstract}
The purpose of this study is to develop a software and hardware complex for robotic defect detection systems, which will reduce the measurement time and reduce labor costs. The main method of detecting defects in this article is the registration of the effect of a change in the resonant frequency of oscillations of rail lashes due to the formation of defects. Simulation and evaluation of the effect of linear dimensions of defects for a regular cylinder and a sphere is given. It is shown that even defects with sizes up to $1 \mathrm{~mm}$ give frequency shifts for the first harmonic up to $10 \mathrm{~Hz}$, and also data are given on the formation of additional oscillatory modes from the position of the defect relative to the edges of the rail lash. The second part of the article contains materials on the development of software and hardware for recording vibration data by a radar method
\end{abstract}

Keywords - robotic system, radar method, defectoscopy, railway rails, frequency of free oscillations, acoustic waves.

\section{INTRODUCTION.}

Currently, for the implementation of robotic flaw detection systems, various systems are used, including both GPR, ultrasound and laser systems [1-4]. But these systems cannot solve the problems of detecting defects at high speed, which leads to an increase in the cost of work and an overall decrease in the efficiency of such systems.

Such systems are relevant for use in railway transport, as used today automation or robotic systems have several disadvantages, such as low productivity of work and a small depth of detection of defects.

Very often, such systems require physical contact with the object of study, which also in turn leads to an even lower effectiveness of such studies.

This article discusses the analysis and development of a radar flaw detector for use in railway transport with the possibility of implementation in a robotic system. The basis for the implementation of such a flaw detector was the effect of changing the frequency of the natural oscillations of the rail due to the formation of a defect in it [5].

Figure 1 shows the general scheme of both the shift of the resonant oscillation frequency due to the formation of a defect in the rail, and the formation of additional vibrational modes.
In earlier studies, the frequency shift was estimated [5]. Here we will try to assess the change in frequency depending on the linear dimensions of the defects in the standard rail of the brand R65. For the calculation we use the formulas given in [6]. Here we estimate only its influence on the shift of the resonant frequencies by the example of a rod fixed at both ends. The following is an assessment of the influence of the linear dimensions of various defects on the resulting frequency shift, depending on the change in the integral density of the rod.

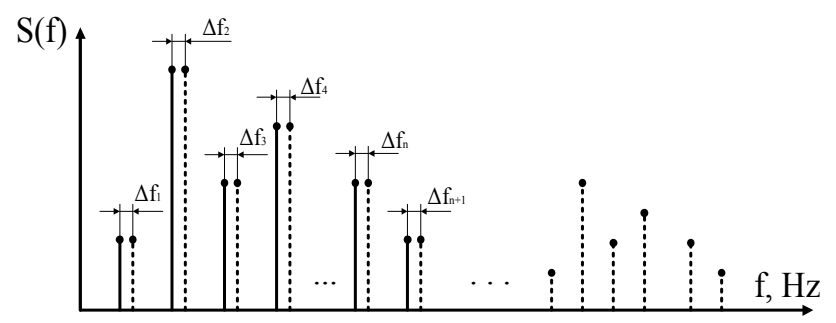

Fig. 1. The principle of revealing a defect by registering additional of vibrational modes in the spectrum of the natural oscillations rail

\section{MATH Modeling}

The evaluation was carried out according to standard formulas for calculating the volume of figures. Here are also graphs of the dependence of the frequency shift on the linear dimensions of the cylinder and the ball cavity, as the two most frequently encountered. It should also be said that since in most cases it is the linear dimensions of the defects that operate.

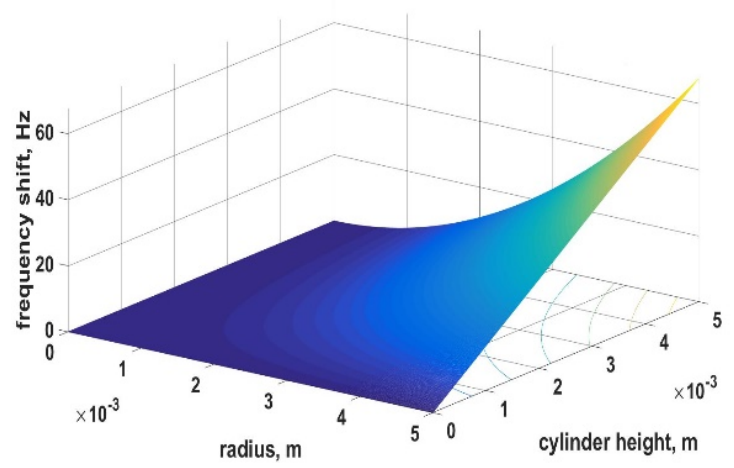

Fig. 2. Dependence of the frequency shift on the size of the defect in the form of a regular cylinder 


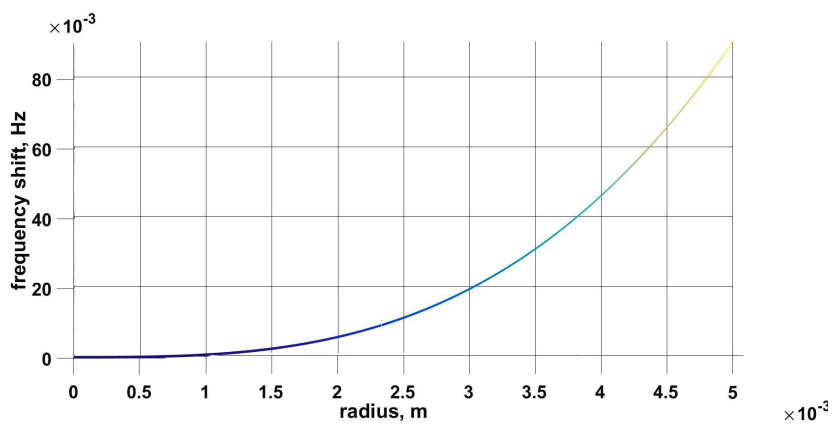

Fig. 3. Dependence of the frequency shift on the defect radius.

As can be seen from the graphs, even small defects with linear dimensions not exceeding a millimeter give the resulting frequency shift up to $10 \mathrm{~Hz}$ for the first harmonic, therefore, registration of such shifts of resonant frequencies with the development of modern element base is not difficult.

Next, we present a mathematical estimate of the dependence of the appearance of additional vibrational modes in a rail due to the formation of a defect in it by the example of the calculation of standing waves depending on the position of the defect in the rail scourge. For ease of calculation, the length of the rail is taken equal to $1 \mathrm{~m}$. The calculation is given by expression (1). We will carry out the calculation for a "cracked" defect, and simulate this defect using an example of gash in a rail of various sizes [7]. The standard formula for calculating the resonant frequencies for a rod fixed from two ends is determined by the formula:

$$
\mathrm{f}=\frac{n}{2 * L} \sqrt{\frac{E}{\rho}}
$$

where, $\mathrm{E}$ is the Young's modulus, $\rho$ is the density of the $\mathrm{P}-65$ rail [8], $\mathrm{L}$ is the rail length, $\mathrm{n}$ is the harmonic number.

Below in Figures 4-5 there are graphs for different widths of the defect and its position in the rail and the calculation of the resulting resonant frequencies generated in the rail. Thus, the appearance of defects in the rail causes the appearance of two additional resonant frequencies along each axis. The frequency in this case will depend on the size of the defect and on its position relative to the edges of the loose scourge.

Defects at the edge of the rail cause the appearance of high resonant frequencies, which may be an additional sign of detection in the case of non-one-digit results of flaw detection by the radar method. The use of this feature will allow more accurate diagnosis compared to using only the frequency shift of the fundamental harmonics of the resonant frequencies [9].

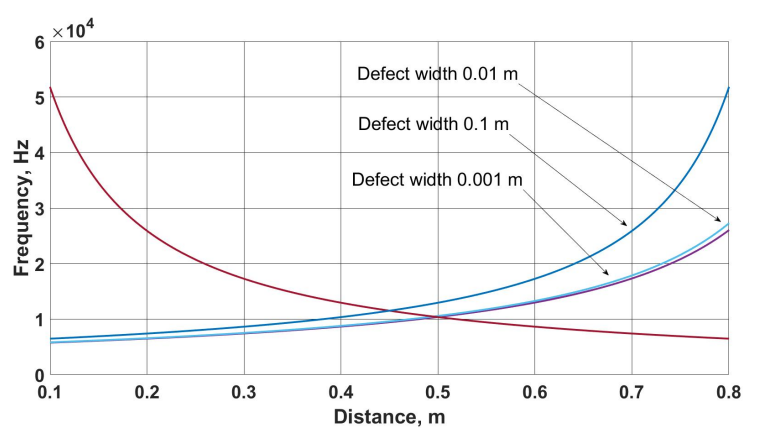

Fig. 4. Dependence of additional resonant frequencies on the position of the defect in the rail along the horizontal axis.

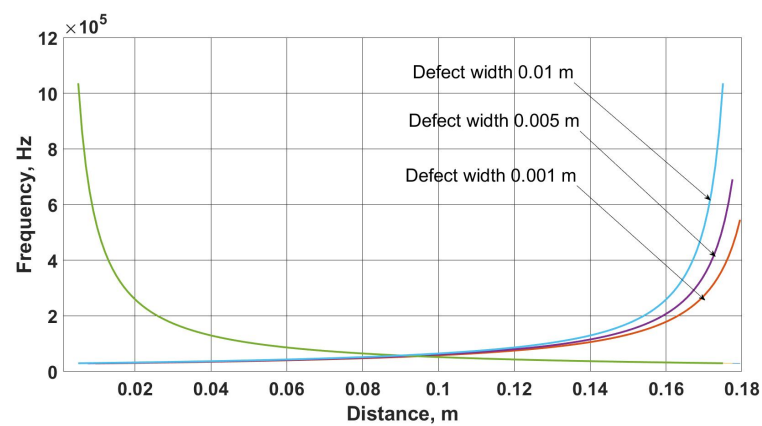

Fig. 5. Dependence of additional resonant frequencies on the position of the defect in the rail vertically.

In real conditions, there will be a picture when there will be a large amount of noise, interference and various oscillatory hormones, which will make it difficult to detect defects. To solve this problem, it is proposed to use the measurement database and compare the rail lash measurements after a certain period of time. Thus, it is possible to register changes in the frequency spectrum in the differential mode and, on its analysis, to decide on the presence of a defect.

\section{Design of the Analog And Digital Part}

To carry out physical modeling and the possibility of recording oscillations, a prototype was developed and created to amplify signals received from a Doppler radar with a frequency of $10 \mathrm{GHz}$. This prototype allows you to amplify signals with an amplitude of $1 \mu \mathrm{V}$ to $1 \mathrm{mV}$. The figure below shows the exploration of the printed circuit board by layers, this board uses a two-channel scheme for the possibility of recording corrections from two sensors in different planes.

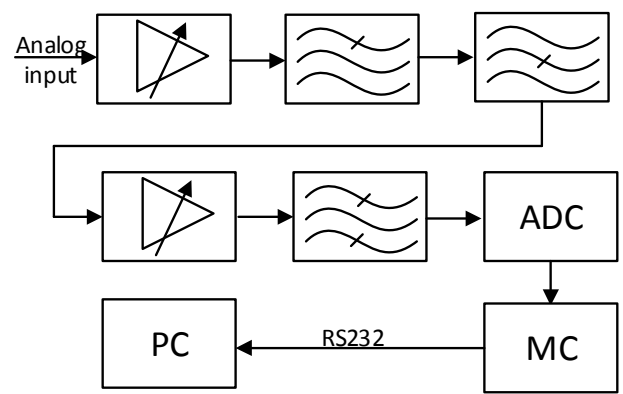

Fig. 6. Block diagram of the printed circuit board 
Analog filtering is carried out using standard active RC filters, as an example the implementation of an active highpass filter is given. The calculation of the nominal and electrical schematic diagram in this article is not given, and is limited only to general issues of the implementation of such systems.

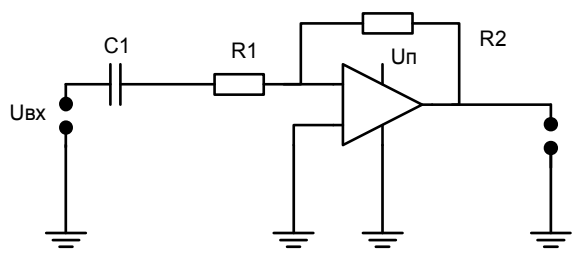

Fig. 7. The implementation scheme of the active high-pass filter

The digital part of this prototype is implemented on the STM32 microcontroller, which allows converting signals into digital form, their subsequent digital filtering, mathematical processing, as well as saving signals in nonvolatile memory, and then transmitting them using a serial interface to a personal computer for further processing.

Below in Figures 8-9, a diluted printed circuit board is shown, as well as the appearance of this board. The dimensions of this board are $10 \times 7 \mathrm{~cm}$, which makes it possible to use it in mobile or compact systems, and the total power consumption does not exceed $50 \mathrm{~mA}$ at a supply voltage of $3 \mathrm{~V}$.

The software part of this layout was developed developed in the Keil software environment.

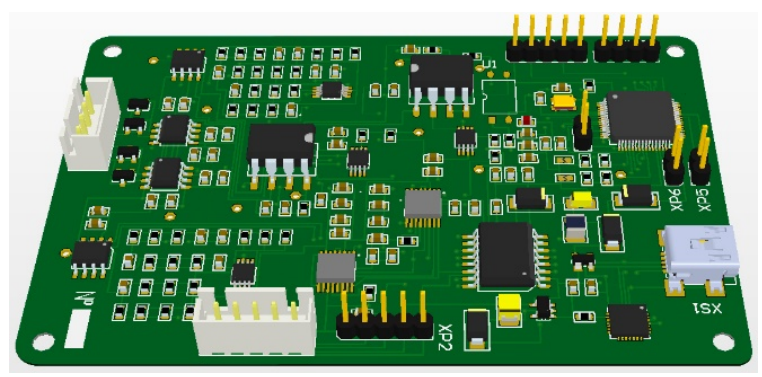

Fig. 8. The appearance of the $\mathrm{PCB}$

The block diagram of the program of the microcontroller is shown in Figure 10.

This block diagram works as follows: when the supply voltage is applied to the board, the main systems and ports are switched on and initialized. Next, the microcontroller goes into standby mode before starting the measurement. When a command comes to the start of the measurement, those values of the frequencies of descritisation, measurement time and other important parameters for each individual measurement are set. Next, the signal is sampled and digitized, stored in non-volatile memory, mathematical calculations and transmission of this data for further processing to a personal computer.

This layout of the board was designed in the Altium Designer software environment, which allows not only to carry out the electrical circuit design, but PCB, with the subsequent preparation of the project for return to production.
To work with this layout, a specialized software product was written in the Matlab software environment. This programmatic implementation will allow a visual assessment of the received signal in real time, as well as in the postprocessing mode, to calculate the fast Fourier transform, wavelet analysis, the construction of the spectrogram.

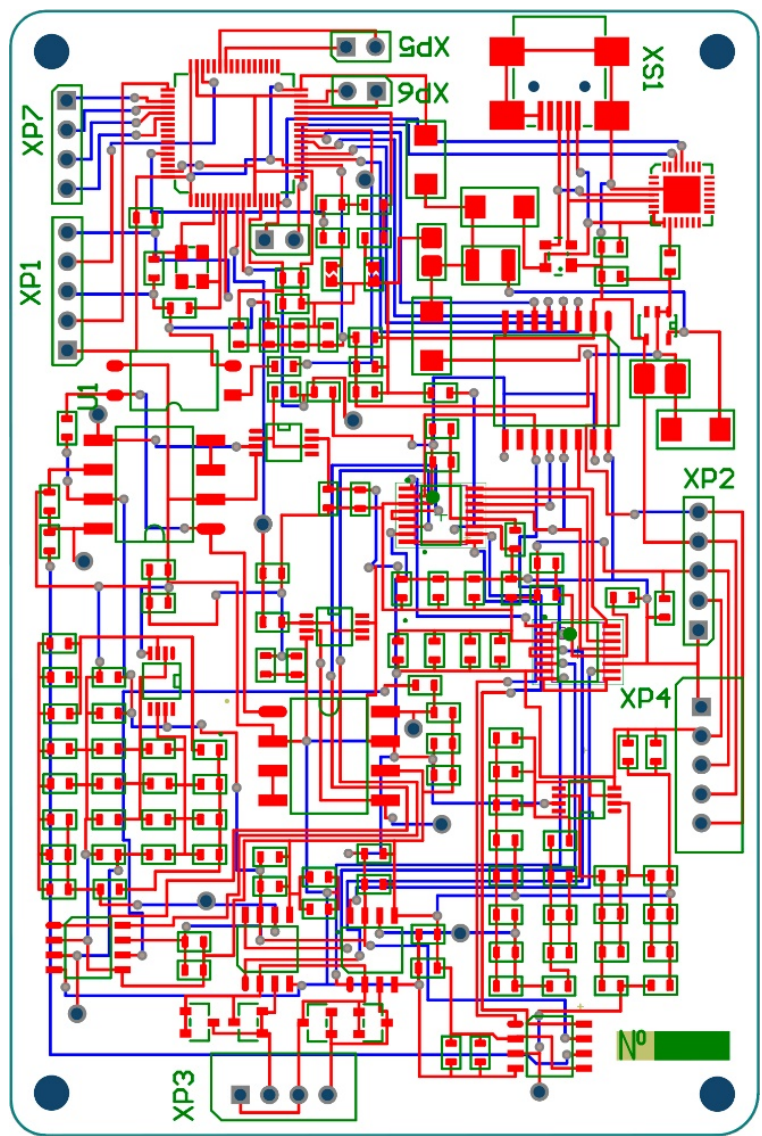

Fig. 9. Designed PCB

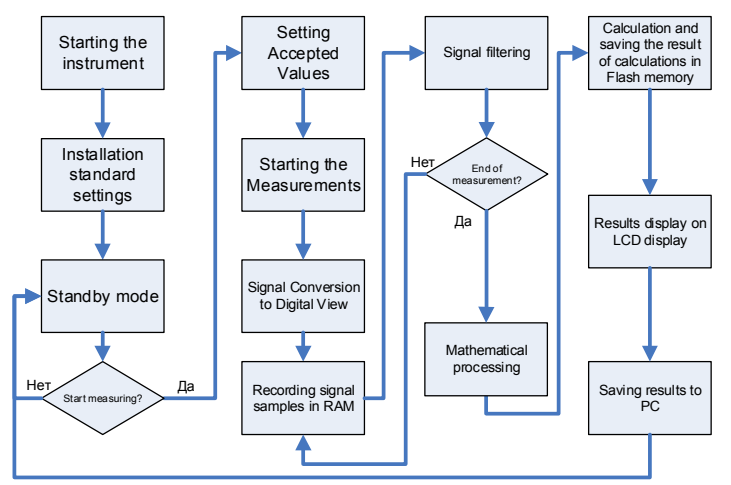

Fig. 10. Block diagram of the microcontroller

Using such a program allows you to find various features of signals in both the time domain and the frequency domain. The calculation of additional digital filters on a personal one allows for more accurate filtering of signals with the suppression of industrial interference. In this case, digital filters with finite impulse response with different number of links are used.

Thus, this program product allows for a complete analysis of the received signals and visualize the results 
obtained by the operator. The appearance of the program is shown in Figure 11.

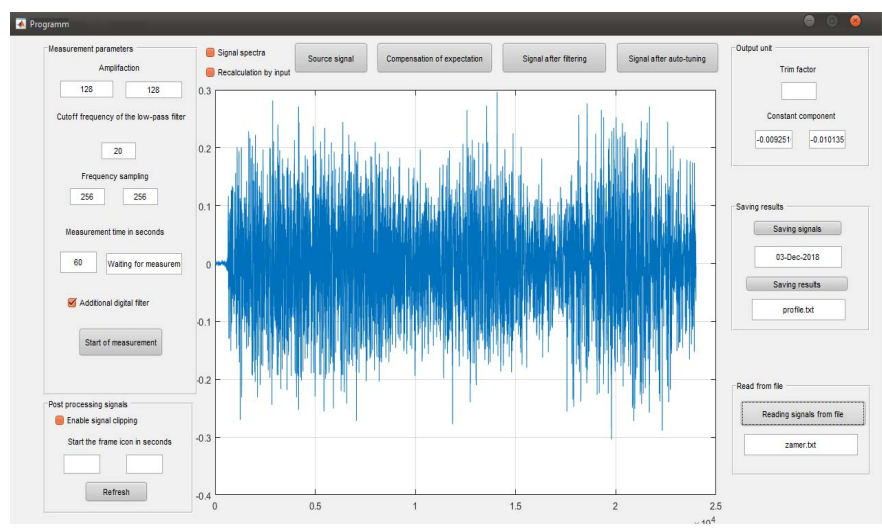

Fig. 11. Appearance of the program for post processing of measurement results

Taking into account all this, it is worth saying that this implementation of the software and hardware complex, developed and created, including for robotic defect search systems, both for the needs of railway transport and for detecting defects in other objects, is a method of registering frequency sweeps in industrial areas. on the modern element base, which will allow in the future with minor modifications in the conditions of industrial operation.

\section{CONCLUSION}

Thus, in this work, it was shown that even minor defects with linear defect sizes (up to $1 \mathrm{~mm}$ ) can introduce significant shifts in the resonant vibration frequencies of rails. Also, the formation of defects leads to the formation of additional resonant frequencies, which can act as an additional feature when defects are detected. The registration of these frequencies by the radar method is often associated with the tasks of recording signals with amplitudes of the order of $1 \mu \mathrm{V}$ and less. In this article, this problem is proposed to be solved with the help of a specialized software and hardware complex, with the possibility of postprocessing the obtained results.

The use of such systems for the needs of non-destructive testing and for specialized analog sensors will reduce labor costs and reduce the cost of their final use in industry. Consequently, the use of such systems will allow you to create a working system that will allow flaw detection or non-destructive testing of rail lashes, such as in real time up to speeds of $100 \mathrm{~km}$ per hour. The use of specialized radar sensors as based on the Dpoler effect or on the basis of recording phase shifts.

\section{ACKNOWLEDGMENT}

The authors thank the Siberian Federal University for providing the infrastructure with the implementation of this project. This work was supported by the Russian Foundation for Basic Research (RFBR) № 16-07-00426.

\section{REFERENCES}

[1] Moustakidis S, Kappatos V, Karlsson P, Selcuk C, Gan T H and Hrissagis K 2014 An Intelligent Methodology for Railways Monitoring Using Ultrasonic Guided Waves. Journal of Nondestructive Evaluation. 33(4) 694-710

[2] Zhao Y, Sun J H, Ma J, Liu S, Guo R, Song J F and Jia Z Q 2014 Insight: Non-Destructive Testing and Condition Monitoring. 56(7) 360-366

[3] hu L, Sun J, Dong M, Li G, Chen J and Niu S 2002 Research of ultrasonic testing applied in rail flaw high-speed. Proceedings of the International Symposium on Precision Mechanical Measurement. (China: Hefei) 1 176-180

[4] Meixedo A, Goncalves A, Calcada, R, Gabriel J, Fonseca H and Martins R 2015 On-line monitoring system for tracks. Exp.at 2015 3rd Experiment International Conference: Online Experimentation (Portugal: University of the Azores Ponta Delgada) 1 133-134

[5] Kudinov D S and Shaidurov G Y 2009 Non-contact nondestructive rail testing, International Siberian Conference on Control and Communications, SIBCON-2009 (Russian Federation: Tomsk) 1 290-295

[6] Shaydurov G Y, Kudinov D S, Kokhonkova E A, Potylitsyn V S, Radar analysis of free oscillations of rail for diagnostics defects, International Conference Information Technologies in Business and Industry 2018 IOP Publishing IOP Conf. Series: Journal of Physics: Conf. Series 1015 (2018) 032182

[7] Tian G Y, Wilson J, Cheng L, Almond D P, Kostson E and Weekes B 2011 Lecture Notes in Electrical Engineering. 96 205-231

[8] Khabarov V N and Demidov V N 2003 Monitoring and diagnostics of the bearing units of track-laying machines. Tyazheloe Mashinostroenie (Russian Federation) 1(10) 33-35 6

[9] Skolnik M I 1976 Radar handbook vol 1: Fundamentals of radiolocation, ed K N Trofymova (Russian Federation) p 456

[10] Skolnik M I 1977 Radar handbook vol 2: Radar Antenna Devices, ed K N Trofymova (Russian Federation) p 408 